\title{
Maximum scattered linear sets and complete caps in Galois spaces*
}

\author{
Daniele Bartoli, Massimo Giulietti, Giuseppe Marino and Olga Polverino
}

\begin{abstract}
Explicit constructions of infinite families of scattered $\mathbb{F}_{q}$-linear sets in $P G\left(r-1, q^{t}\right)$ of maximal rank $\frac{r t}{2}$, for $t$ even, are provided. When $q=2$ and $r$ is odd, these linear sets correspond to complete caps in $A G\left(r, 2^{t}\right)$ fixed by a translation group of size $2^{\frac{r t}{2}}$. The doubling construction applied to such caps gives complete caps in $A G\left(r+1,2^{t}\right)$ of size $2^{\frac{r t}{2}+1}$. For Galois spaces of even dimension greater than 2 and even square order, this solves the long-standing problem of establishing whether the theoretical lower bound for the size of a complete cap is substantially sharp.
\end{abstract}

Keywords: Galois spaces, linear sets, complete caps.

\section{Introduction}

Let $\Lambda=P G\left(V, \mathbb{F}_{q^{t}}\right)=P G\left(r-1, q^{t}\right), q=p^{h}, p$ prime, with $V$ vector space of dimension $\mathrm{r}$ over $\mathbb{F}_{q^{t}}$, and let $L$ be a set of points of $\Lambda$. The set $L$ is said to be an $\mathbb{F}_{q}$-linear set of $\Lambda$ of rank $t$ if

${ }^{*}$ The research was supported by Ministry for Education, University and Research of Italy MIUR (Project PRIN 2012 "Geometrie di Galois e strutture di incidenza") and by the Italian National Group for Algebraic and Geometric Structures and their Applications (GNSAGA - INdAM) 
it is defined by the non-zero vectors of an $\mathbb{F}_{q}$-vector subspace $U$ of $V$ of dimension $t$, i.e.

$$
L=L_{U}=\left\{\langle\mathbf{u}\rangle_{\mathbb{F}_{q^{t}}}: \mathbf{u} \in U \backslash\{\mathbf{0}\}\right\}
$$

We point out that different vector subspaces can define the same linear set. For this reason a linear set and the vector space defining it must be considered as coming in pair.

Let $\Omega=P G\left(W, \mathbb{F}_{q^{t}}\right)$ be a subspace of $\Lambda$ and let $L_{U}$ be an $\mathbb{F}_{q^{-}}$-linear set of $\Lambda$. Then $\Omega \cap L_{U}$ is an $\mathbb{F}_{q}$-linear set of $\Omega$ defined by the $\mathbb{F}_{q}$-vector subspace $U \cap W$ and, if $\operatorname{dim}_{\mathbb{F}_{q}}(W \cap U)=i$, we say that $\Omega$ has weight $i$ in $L_{U}$. Hence a point of $\Lambda$ belongs to $L_{U}$ if and only if it has weight at least 1 and if $L_{U}$ has rank $k$, then $\left|L_{U}\right| \leq q^{k-1}+q^{k-2}+\cdots+q+1$. For further details on linear sets see [18], [11], [12], [13], [14].

An $\mathbb{F}_{q}$-linear set $L_{U}$ of $\Lambda$ of rank $k$ is scattered if all of its points have weight 1 , or equivalently, if $L_{U}$ has maximum size $q^{k-1}+q^{k-2}+\cdots+q+1$. A scattered $\mathbb{F}_{q}$-linear set of $\Lambda$ of highest possible rank is a maximum scattered $\mathbb{F}_{q}$-linear set of $\Lambda$; see [3].

In 3] the authors obtain the following result on the rank of a maximum scattered linear set; see also [9].

Theorem 1.1. [3, Thms 2.1, 4.3 and 4.2]) If $L_{U}$ is a maximum scattered $\mathbb{F}_{q}$-linear set of $P G\left(r-1, q^{t}\right)$ of rank $k$, then

$$
\begin{aligned}
k & =\frac{r t}{2} \quad \text { if } r \text { is even, } \\
\frac{r t-t}{2} & \leq k \leq \frac{r t}{2} \quad \text { if } r \text { is odd. }
\end{aligned}
$$

Also, if $r t$ is even and $L_{U}$ is a maximum scattered $\mathbb{F}_{q}$-linear set of $P G\left(r-1, q^{t}\right)$ of rank $\frac{r t}{2}$, then $L_{U}$ is a two-intersection set (with respect to hyperplanes) in $P G\left(r-1, q^{t}\right)$ with intersection numbers $\theta_{\frac{r t}{2}-t-1}(q)=\frac{q^{\frac{r t}{2}-t}-1}{q-1}$ and $\theta_{\frac{r t}{2}-t}(q)=\frac{q^{\frac{r t}{2}-t+1}}{q-1}$.

When $r$ is even there always exists an $\mathbb{F}_{q}$-scattered linear set of rank $\frac{r t}{2}$ in $P G\left(r-1, q^{t}\right)$ (see [9, Theorem 2.5.5] for an explicit example) whereas, when $r$ is odd, the upper bound $\frac{r t}{2}$ is 
attained in the following cases:

- $r=3, t=2$ (Baer subplanes),

- $r=3, t=4[2$, Section 3]),

- $r>3, t=2, q=2$ [3, Thm. 4.4]),

- $r \geq 3,(t-1) \mid r(t$ even), $q>2[3$, Thm. 4.4]).

This means that, for a given value of $r$, examples of maximum scattered linear sets have been shown to exist only for a small number of $t$ 's. It should be also noted that, differently from what happens for $r$ even, in the case $r$ odd the proof of Theorem 4.4 in [3] shows the existence of such maximum scattered linear sets without giving explicit examples.

In the first part of this paper we construct three different families of scattered $\mathbb{F}_{q}$-linear sets in $P G\left(2, q^{t}\right), t \geq 4$ even, of rank $\frac{3 t}{2}$, for infinite values of the prime power $q$. This allows us to produce for each integer $r \geq 5$, scattered $\mathbb{F}_{q}$-linear sets in $P G\left(r-1, q^{t}\right)$ of rank $\frac{r t}{2}(t$ even). More precisely we show that

Theorem 1.2. There exist examples of scattered $\mathbb{F}_{q}$-linear sets in $P G\left(r-1, q^{t}\right)$, $t$ even, of rank $\frac{r t}{2}$ in the following cases:

- $q=2$ and $t \geq 4$;

- $q \geq 2$ and $t \not \equiv 0 \bmod 3$;

- $q \equiv 1 \bmod 3$ and $t \equiv 0 \bmod 3$.

In the second part of the paper we point out the relationship between maximum scattered linear sets and complete caps in affine spaces over finite fields of even characteristic. A cap in an affine or projective Galois space is a set of points no three of which collinear; a cap which is maximal with respect to set-theoretical inclusion is said to be complete. A long-standing issue 
in Finite Geometry is to ask for explicit constructions of small complete caps in Galois spaces. The trivial lower bound for the size of a complete cap in a Galois space of dimension $n$ and order $q$ is

$$
\sqrt{2} \cdot \sqrt{q}^{n-1}
$$

If $q$ is even and $n$ is odd, such bound is substantially sharp: the existence of a complete cap of size $3 q+2$ in $P G(3, q)$ was showed by Segre [19], whose construction was later generalized by Pambianco and Storme [17] to complete caps of size $2 q^{s}$ in $A G(2 s+1, q)$. Otherwise, all known infinite families of complete caps have size far from (10); see the survey paper [7]. Here we prove that (11) is essentially sharp also when $n \geq 4$ is even, provided that $q$ is an even square.

Theorem 1.3. Let $q=2^{t}, t$ even, and $n \geq 4$ even. Then there exists a complete cap in $A G(n, q)$ of size $2 \sqrt{q}^{n-1}$.

Theorem 1.3 relies on the fact that $\mathbb{F}_{2}$-linear sets in $P G\left(r-1,2^{t}\right)$ of maximal rank $\frac{r t}{2}$, for $t$ even and $r$ odd, naturally correspond to complete caps in $A G\left(r, 2^{t}\right)$ fixed by a translation group of size $2^{\frac{r t}{2}}$. Then the scattered $\mathbb{F}_{2}$-linear sets of maximal rank described in this paper, together with the doubling construction for translation caps as described in [6], provide complete caps in $A G(r+1, q)$ of size $2 q^{\frac{r}{2}}$, for $q=2^{t}$. We point out that complete caps in the projective space $P G(r+1, q)$ with size of the same order of magnitude can also be constructed (see Remark 4.8).

\section{Constructions of maximum scattered linear sets in $P G\left(2, q^{2 n}\right)$}

In this section we want to construct infinite families of scattered $\mathbb{F}_{q}$-linear sets of rank $3 n$ in the projective plane $P G\left(2, q^{2 n}\right)$, with $n \geq 2$. Note that, by Theorem 1.1, such scattered linear sets are two intersection sets (with respect to the lines) of the plane.

Consider the finite field $\mathbb{F}_{q^{6 n}}$ as a 3 -dimensional vector space over its subfield $\mathbb{F}_{q^{2 n}}, n \geq 2$, and let $\mathbb{P}=P G\left(\mathbb{F}_{q^{6 n}}, \mathbb{F}_{q^{2 n}}\right)=P G\left(2, q^{2 n}\right)$ be the associated projective plane. 
The following proposition can be easily verified.

Proposition 2.1. Let $f: \mathbb{F}_{q^{3 n}} \rightarrow \mathbb{F}_{q^{3 n}}$ be an $\mathbb{F}_{q}$-linear map, $\omega$ an element of $\mathbb{F}_{q^{2 n}} \backslash \mathbb{F}_{q^{n}}$ and consider the subset of $\mathbb{F}_{q^{6 n}}$

$$
U=\left\{f(x)+x \omega: x \in \mathbb{F}_{q^{3 n}}\right\}
$$

Then, the set

$$
L_{U}=\left\{\langle f(x)+x \omega\rangle_{\mathbb{F}_{q^{2 n}}}: x \in \mathbb{F}_{q^{3 n}}^{*}\right\}
$$

is an $\mathbb{F}_{q}$-linear of rank $3 n$ of the projective plane $\mathbb{P}=P G\left(2, q^{2 n}\right)$. Also, put

$$
Q_{f}:=\left\{\frac{f(x)+x \omega}{f(y)+y \omega}: x, y \in \mathbb{F}_{q^{3 n}}, y \neq 0\right\},
$$

the set $L_{U}$ turns out to be scattered if and only if $Q_{f} \cap \mathbb{F}_{q^{2 n}}=\mathbb{F}_{q}$.

Proof. We first observe that $\{1, \omega\}$ is an $\mathbb{F}_{q^{n}}$-basis of $\mathbb{F}_{q^{2 n}}$ and an $\mathbb{F}_{q^{3 n}}$-basis of $\mathbb{F}_{q^{6 n}}$, as well. Also, since $f$ is an $\mathbb{F}_{q}$-linear map, the subset $U=\left\{f(x)+x \omega: x \in \mathbb{F}_{q^{3 n}}\right\}$ of $\mathbb{F}_{q^{6 n}}$ is closed under addition and $\mathbb{F}_{q}$-scalar multiplication, and hence it is an $\mathbb{F}_{q}$-vector subspace of $\mathbb{F}_{q^{6 n}}$. This means that the set $L_{U}$ turns out to be an $\mathbb{F}_{q}$-linear set of rank $3 n$ of the plane $\mathbb{P}$. Also, $L_{U}$ is not scattered if and only if there exists a point $P_{x}:=\langle f(x)+x \omega\rangle_{\mathbb{F}_{q^{2 n}}}$ of $L_{U}$, with $x \in \mathbb{F}_{q^{3 n}}^{*}$, having weight grater than 1 , and hence there exist $y \in \mathbb{F}_{q^{3 n}}^{*}$ and $\lambda \in \mathbb{F}_{q^{2 n}} \backslash \mathbb{F}_{q}$ such that

$$
f(x)+x \omega=\lambda(f(y)+y \omega) .
$$

The assertion follows.

Let now

$$
\omega^{2}=A+B \omega
$$

with $A, B \in \mathbb{F}_{q^{n}}$ and $A \neq 0$, and suppose that there exist $x, y \in \mathbb{F}_{q^{3 n}}^{*}$ and $\lambda \in \mathbb{F}_{q^{2 n}} \backslash \mathbb{F}_{q}$ satisfying Equation (3). Such an equation implies that

$$
\left(\frac{f(x)+x \omega}{f(y)+y \omega}\right)^{q^{2 n}}=\frac{f(x)+x \omega}{f(y)+y \omega}
$$


i.e., taking (44) into account, we get

$$
\begin{aligned}
& f(x)^{q^{2 n}} f(y)+x^{q^{2 n}} y A+\left(f(x)^{q^{2 n}} y+f(y) x^{q^{2 n}}+x^{q^{2 n}} y B\right) \omega= \\
& =f(y)^{q^{2 n}} f(x)+y^{q^{2 n}} x A+\left(f(y)^{q^{2 n}} x+f(x) y^{q^{2 n}}+y^{q^{2 n}} x B\right) \omega .
\end{aligned}
$$

Since $\{1, \omega\}$ is an $\mathbb{F}_{q^{3 n}}$-basis of $\mathbb{F}_{q^{6 n}}$, the above equality is equivalent to

$$
\left\{\begin{array}{l}
f(x)^{q^{2 n}} f(y)-f(y)^{q^{2 n}} f(x)=\left(x y^{q^{2 n}}-y x^{q^{2 n}}\right) A \\
f(x)^{q^{2 n}} y+f(y) x^{q^{2 n}}-f(y)^{q^{2 n}} x-f(x) y^{q^{2 n}}=\left(x y^{q^{2 n}}-y x^{q^{2 n}}\right) B
\end{array} .\right.
$$

The previous arguments allow us to reformulate the previous proposition in the following way which will be useful in the sequel.

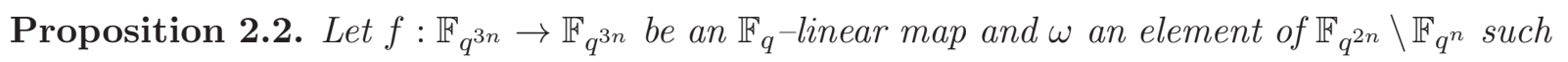
that $\omega^{2}=A+B \omega$, with $A, B \in \mathbb{F}_{q^{n}}$ and $A \neq 0$. The set

$$
L_{U}=\left\{\langle f(x)+x \omega\rangle_{\mathbb{F}_{q^{2 n}}}: x \in \mathbb{F}_{q^{3 n}}^{*}\right\}
$$

turns out to be a scattered $\mathbb{F}_{q}$-linear of rank $3 n$ of the projective plane $\mathbb{P}=P G\left(2, q^{2 n}\right)$ if and only if for each pair $(x, y) \in \mathbb{F}_{q^{3 n}}^{*} \times \mathbb{F}_{q^{3 n}}^{*}$ satisfying the following equations

$$
\begin{gathered}
f(x)^{q^{2 n}} f(y)-f(y)^{q^{2 n}} f(x)=\left(x y^{q^{2 n}}-y x^{q^{2 n}}\right) A \\
f(x)^{q^{2 n}} y+f(y) x^{q^{2 n}}-f(y)^{q^{2 n}} x-f(x) y^{q^{2 n}}=\left(x y^{q^{2 n}}-y x^{q^{2 n}}\right) B,
\end{gathered}
$$

the quotient

$$
\lambda:=\frac{f(x)+x \omega}{f(y)+y \omega}
$$

is an element of $\mathbb{F}_{q}^{*}$.

In the sequel we will exhibit examples of $\mathbb{F}_{q}$-linear maps of $\mathbb{F}_{q^{3 n}}$ satisfying the previous properties. In particular, we face with the monomial and the binomial cases. 
Monomial case: $f(x):=a x^{q^{i}}, a \in \mathbb{F}_{q^{3 n}}^{*}$ and $1 \leq i \leq 3 n-1$

In such a case we first show that for any value of $q \geq 2$, under suitable assumptions on $a \in \mathbb{F}_{q^{3 n}}^{*}$ and on the integers $i$ and $n$, we get a scattered $\mathbb{F}_{q}$-linear set of the projective plane $P G\left(2, q^{2 n}\right)$ of rank $3 n$. Denoting by $N_{q^{3 n} / q^{3}}(\cdot)$ the norm function from $\mathbb{F}_{q^{3 n}}$ over $\mathbb{F}_{q^{3}}$, we have the following

Theorem 2.3. For any prime power $q \geq 2$ and any integer $n \not \equiv 0 \bmod 3$, the set

$$
L_{U}=\left\{\left\langle a x^{q^{i}}+x \omega\right\rangle_{\mathbb{F}_{q^{2 n}}}: x \in \mathbb{F}_{q^{3 n}}^{*}\right\}
$$

satisfying the following assumptions:

(i) $\operatorname{gcd}(i, 2 n)=1$ and $\operatorname{gcd}(i, 3 n)=3$

(ii) $\quad N_{q^{3 n} / q^{3}}(a) \notin \mathbb{F}_{q}$

is a scattered $\mathbb{F}_{q}$-linear set of the projective plane $P G\left(2, q^{2 n}\right)$ of rank $3 n$.

Proof. By Proposition 2.2, in order to prove the statement we have first to determine the solutions $x, y \in \mathbb{F}_{q^{3 n}}^{*}$ of Equations (5) and ([6), where we have chosen $f(x)=a x^{q^{i}}$, with $a \in \mathbb{F}_{q^{3 n}}^{*}$ and $1 \leq i \leq 3 n-1$ and satisfying Conditions $(i)$ and $(i i)$. With these assumptions, Equations (5) and (6) become

$$
a^{q^{2 n}+1}\left(x^{q^{2 n}} y-x y^{q^{2 n}}\right)^{q^{i}}=\left(x y^{q^{2 n}}-y x^{q^{2 n}}\right) A
$$

and

$$
a^{q^{2 n}}\left(x^{q^{2 n+i}} y-x y^{q^{2 n+i}}\right)+a\left(x^{q^{2 n}} y^{q^{i}}-x^{q^{i}} y^{q^{2 n}}\right)=\left(x y^{q^{2 n}}-y x^{q^{2 n}}\right) B
$$

Let $s:=x y^{q^{2 n}}-y x^{q^{2 n}}$. By (8), if $s \neq 0$, then $s$ turns out to be a solution in $\mathbb{F}_{q^{3 n}}$ of the equation

$$
z^{q^{i}-1}=-\frac{A}{a^{q^{2 n}+1}}
$$


and, from Conditions (i), Equation (10) has solutions if and only if $N_{q^{3 n} / q^{3}}\left(-\frac{A}{a^{q^{2 n}+1}}\right)=1$, namely

$$
(-1)^{n} N_{q^{3 n} / q^{3}}(A)=\left(N_{q^{3 n} / q^{3}}(a)\right)^{q+1} \quad \text { if } 2 n \equiv 1 \bmod 3
$$

or

$$
(-1)^{n} N_{q^{3 n} / q^{3}}(A)=\left(N_{q^{3 n} / q^{3}}(a)\right)^{q^{2}+1} \quad \text { if } 2 n \equiv-1 \bmod 3 .
$$

Since $A \in \mathbb{F}_{q^{n}}^{*}$ and since $n \not \equiv 0 \bmod 3$, we get $N_{q^{3 n} / q^{3}}(A) \in \mathbb{F}_{q}$ and from Condition $(i i)$ it follows that both Equations (11) and (12) cannot be satisfied. This means that $s=0$ and hence $x=\alpha y$, for some $\alpha \in \mathbb{F}_{q^{n}}^{*}$. Substituting in (9), we get

$$
\left(\alpha^{q^{i}}-\alpha\right)\left(a^{q^{2 n}} y^{q^{2 n+i}+1}-a y^{q^{2 n}+q^{i}}\right)=0 .
$$

If $\alpha^{q^{i}} \neq \alpha$, raising the previous equation to the $q^{n}-$ th power, then

$$
y^{\left(q^{n}-1\right)\left(q^{i}-1\right)}=a^{1-q^{n}}
$$

i.e.

$$
\left(a y^{q^{i}-1}\right)^{q^{n}-1}=1
$$

which is verified if and only if $y^{q^{i}-1}=\frac{\beta}{a}$, for some $\beta \in \mathbb{F}_{q^{n}}^{*}$. This means that $y \in \mathbb{F}_{q^{3 n}}^{*}$ turns out to be a solution of the equation $z^{q^{i}-1}=\beta / a$ and, from Conditions $(i)$, this happens if and only if

$$
N_{q^{3 n} / q^{3}}(\beta)=N_{q^{3 n} / q^{3}}(a)
$$

Since $\beta \in \mathbb{F}_{q^{n}}$, from Conditions $(i)$ it follows $N_{q^{3 n} / q^{3}}(\beta) \in \mathbb{F}_{q}^{*}$ and taking Condition (ii) into account, we get a contradiction. Hence the element $\alpha \in \mathbb{F}_{q^{n}}$ is such that $\alpha^{q^{i}}=\alpha$, and since $\operatorname{gcd}(i, n)=1$, we get $\alpha \in \mathbb{F}_{q}^{*}$. Substituting $x=\alpha y$ in (7) we get $\lambda=\alpha \in \mathbb{F}_{q}^{*}$, proving the assertion by Proposition 2.2

Observe that the $3 n$-dimensional $\mathbb{F}_{q}$-vector subspace $U$ of $F_{q^{6 n}}$ defining the linear set $L_{U}$ of Theorem 2.3 is also an $n$-dimensional $\mathbb{F}_{q^{3}}$-vector subspace. In particular, when $n=2, U$ is 
a 2-dimensional $\mathbb{F}_{q^{3}}$-subspace of $\mathbb{F}_{q^{12}}$ and hence it can be always seen as the set of zeros of a polynomial

$$
x^{q^{6}}+\alpha x^{q^{3}}+\beta x \in \mathbb{F}_{q^{12}}[x],
$$

where $N_{q^{12} / q^{3}}(\beta)=1$ and $\alpha^{q^{3}+1}=\beta^{q^{3}}-\beta^{q^{6}+q^{3}+1}$; see [1]. Hence, the examples of scattered $\mathbb{F}_{q^{-}}$ linear sets of rank 6 constructed in $P G\left(2, q^{4}\right)$ by [2] belong to the family presented in Theorem 2.3.

Now, we will construct, for $q \equiv 1 \bmod 3$, another family of scattered $\mathbb{F}_{q}$-linear sets of $P G\left(2, q^{2 n}\right)$ of rank $3 n$ defined by an $\mathbb{F}_{q^{-}}$-vector subspace which is not an $\mathbb{F}_{q^{3}}$-subspace. Indeed,

Theorem 2.4. For any prime power $q \equiv 1 \bmod 3$ and any integer $n \geq 2$, the set

$$
L_{U}=\left\{\left\langle a x^{q^{i}}+x \omega\right\rangle_{\mathbb{F}_{q^{2 n}}}: x \in \mathbb{F}_{q^{3 n}}^{*}\right\}
$$

satisfying the following assumptions

(I) $\operatorname{gcd}(i, 2 n)=\operatorname{gcd}(i, 3 n)=1$,

$(I I) \quad\left(N_{q^{3 n} / q}(a)\right)^{\frac{q-1}{3}} \neq 1$

is a scattered $\mathbb{F}_{q}$-linear set of the projective plane $P G\left(2, q^{2 n}\right)$ of rank $3 n$.

Proof. The first part of the proof is the same as in Theorem 2.3. So, we have to determine the solutions $x, y \in \mathbb{F}_{q^{3 n}}^{*}$ of Equations (8) and (9). Putting again $s:=x y^{q^{2 n}}-y x^{q^{2 n}}$, if $s \neq 0$, from the previous equality, $s$ turns out to be a solution in $\mathbb{F}_{q^{3 n}}^{*}$ of (10) and, from Conditions $(I)$. Equation (10) has solutions if and only if $N_{q^{3 n} / q}\left(-\frac{A}{a^{q^{2 n}}+1}\right)=1$, namely

$$
\left(N_{q^{3 n} / q}(a)\right)^{2}=(-1)^{n}\left(N_{q^{n} / q}(A)\right)^{3},
$$

implying

$$
\left(\left(N_{q^{3 n} / q}(a)\right)^{\frac{q-1}{3}}\right)^{2}=(-1)^{\frac{n(q-1)}{3}} .
$$


If $\frac{n(q-1)}{3}$ is even, Condition $(I I)$ implies that $q$ is odd and $\left(N_{q^{3 n} / q}(a)\right)^{\frac{q-1}{3}}=-1$, and raising this equality to the $3-$ rd power we get a contradiction. If $\frac{n(q-1)}{3}$ is odd, then $q$ is even, and hence $\left(N_{q^{3 n} / q}(a)\right)^{\frac{q-1}{3}}=1$, again contradicting Condition $(I I)$. This means that $s=0$ and hence $x=\alpha y$, for some $\alpha \in \mathbb{F}_{q^{n}}^{*}$, and arguing again as in the previous proof, if $\alpha^{q^{i}} \neq \alpha$, then $y \in \mathbb{F}_{q^{3 n}}^{*}$ turns out to be a solution of the equation $z^{q^{i}-1}=\beta / a$, for some $\beta \in \mathbb{F}_{q^{*}}^{*}$. From Conditions $(I)$, this happens if and only if

$$
\left(N_{q^{n} / q}(\beta)\right)^{3}=N_{q^{3 n} / q}(a)
$$

which means that $N_{q^{n} / q}(\beta)$ is a solution in $\mathbb{F}_{q}^{*}$ of the equation $z^{3}=N_{q^{3 n} / q}(a)$, contradicting Condition $(I I)$. Hence the element $\alpha \in \mathbb{F}_{q^{n}}^{*}$ is such that $\alpha^{q^{i}}=\alpha$, and since $\operatorname{gcd}(i, n)=1$, we get $\alpha \in \mathbb{F}_{q}^{*}$, yielding as in the previous proof $\lambda \in \mathbb{F}_{q}^{*}$. By Proposition 2.2, we have the assertion.

Putting together Theorems 2.3 and 2.4 we get the following

Theorem 2.5. - If $n \not \equiv 0 \bmod 3$, there exist scattered $\mathbb{F}_{q}$-linear sets in $P G\left(2, q^{2 n}\right)$ of rank $3 n$ for each prime power $q \geq 2$.

- If $n \equiv 0 \bmod 3$, there exist scattered $\mathbb{F}_{q}$-linear sets in $P G\left(2, q^{2 n}\right)$ of rank $3 n$ for each prime power $q \equiv 1 \bmod 3$.

Binomial case: $f(x):=a x^{q^{i}}+b y^{q^{j}}, a, b \in \mathbb{F}_{q^{3 n}}^{*}$ and $1 \leq i, j \leq 3 n-1$

With this type of function it is clear that the linear set (21) has $\mathbb{F}_{q}$ as maximum subfield of linearity when $\operatorname{gcd}(i, j, 2 n)=1$. In particular we will study the case when $j=2 n+i$ and, obviously $\operatorname{gcd}(i, 2 n)=1$. First of all we need a technical lemma. Denoting by $\operatorname{Tr}_{q^{3 n} / q}(\cdot)$ the trace function from $\mathbb{F}_{q^{3 n}}$ over $\mathbb{F}_{q}$, we can consider the non-degenerate symmetric bilinear form of $\mathbb{F}_{q^{3 n}}$ over $\mathbb{F}_{q}$ defined by the following rule $\left\langle x, y>:=T r_{q^{3 n} / q}(x y)\right.$. Then the adjoint map $\bar{\varphi}$ 
of an $\mathbb{F}_{q}$-linear map $\varphi(x)=\sum_{i=0}^{3 n-1} a_{i} x^{q^{i}}$ of $\mathbb{F}_{q^{3 n}}$ is $\bar{\varphi}(x)=\sum_{i=0}^{3 n-1} a_{i}^{q^{3 n-i}} x^{q^{3 n-i}}$ (see e.g. [15, Sec. $2.2])$. Now, we can prove the following

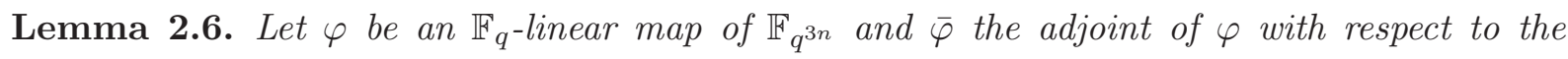
bilinear form $<,>$. Then the maps defined by $\varphi(x) / x$ and $\bar{\varphi}(x) / x$ have the same image.

Proof. Let $\mathbb{V}=\mathbb{F}_{q^{3 n}} \times \mathbb{F}_{q^{3 n}}$ and let $\sigma: \mathbb{V} \times \mathbb{V} \longrightarrow \mathbb{F}_{q^{3 n}}$ be the non-degenerate alternating bilinear form of $\mathbb{V}$ defined by $\sigma((x, y),(u, v))=x v-y u$. Then

$$
\sigma^{\prime}((x, y),(u, v))=\operatorname{Tr}_{q^{3 n} / q}(\sigma((x, y),(u, v)))
$$

is a non-degenerate alternating bilinear form on $\mathbb{V}$, when $\mathbb{V}$ is regarded as a $6 n$-dimensional vector space over $\mathbb{F}_{q}$. Let $\perp$ and $\perp^{\prime}$ be the orthogonal complement maps defined by $\sigma$ and $\sigma^{\prime}$

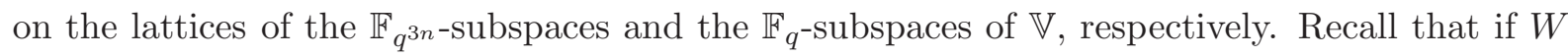

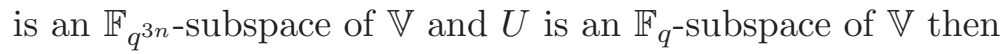

$$
\operatorname{dim}_{\mathbb{F}_{q^{3 n}}} W^{\perp}+\operatorname{dim}_{\mathbb{F}_{q^{3 n}}} W=2
$$

and

$$
\operatorname{dim}_{\mathbb{F}_{q}} U^{\perp^{\prime}}+\operatorname{dim}_{\mathbb{F}_{q}} U=6 n .
$$

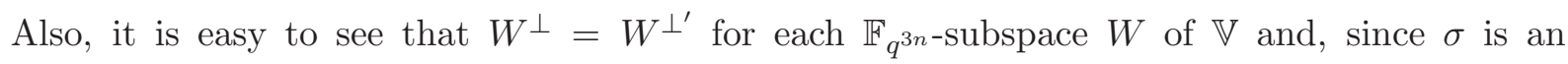

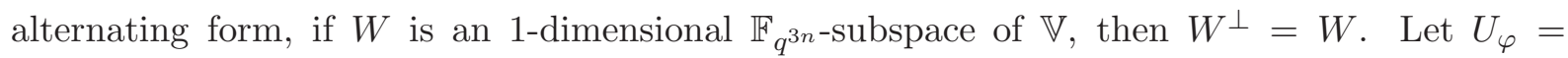
$\left\{(x, \varphi(x)): x \in F_{q^{3 n}}\right\}$, where $\varphi$ is an $\mathbb{F}_{q^{-}}$-linear map of $\mathbb{F}_{q^{3 n}}$. Then $U_{\varphi}$ is a $3 n$-dimensional $\mathbb{F}_{q^{-}}$-subspace of $\mathbb{V}$ and a direct calculation shows that $U_{\varphi}^{\perp^{\prime}}=U_{\bar{\varphi}}$. Note that an element $t \in \mathbb{F}_{q^{3 n}}$ belongs to the image of the map $\varphi(x) / x$ if and only if the point $P_{t}=\langle(1, t)\rangle_{\mathbb{F}^{3 n}}$ of $P G\left(\mathbb{V}, \mathbb{F}_{q^{3 n}}\right)=$ $P G\left(1, q^{3 n}\right)$ belongs to the $\mathbb{F}_{q}$-linear set $L_{U_{\varphi}}$. Since $P_{t}^{\perp}=P_{t}^{\perp^{\prime}}=P_{t}$, by using the Grassmann formula, we get

$$
P_{t} \in L_{U_{\varphi}} \Leftrightarrow \operatorname{dim}_{\mathbb{F}_{q}}\left(U_{\varphi} \cap P_{t}\right) \geq 1 \Leftrightarrow \operatorname{dim}_{\mathbb{F}_{q}}\left(U_{\varphi}^{\perp^{\prime}} \cap P_{t}^{\perp^{\prime}}\right) \geq 1 \Leftrightarrow \operatorname{dim}_{\mathbb{F}_{q}}\left(U_{\bar{\varphi}} \cap P_{t}\right) \geq 1 \Leftrightarrow P_{t} \in L_{U_{\bar{\varphi}}},
$$


i.e., $t \in \mathbb{F}_{q^{3 n}}$ belongs to the image of the map $\varphi(x) / x$ if and only if $t$ belongs to the image of the $\operatorname{map} \bar{\varphi}(x) / x$.

Now we can show the following result.

Proposition 2.7. Let $f:=f_{i, a, b}: x \in \mathbb{F}_{q^{3 n}} \rightarrow a x^{q^{i}}+b x^{q^{2 n+i}} \in \mathbb{F}_{q^{3 n}}$, with $a, b \in \mathbb{F}_{q^{3 n}}^{*}$ and $\operatorname{gcd}(i, 2 n)=1$, and let $\omega$ be an element of $\mathbb{F}_{q^{2 n}} \backslash \mathbb{F}_{q^{n}}$ such that $\omega^{2}=A+B \omega$, with $A, B \in \mathbb{F}_{q^{n}}$ and $A \neq 0$. If

$$
\frac{f_{i, a, b}(x)}{x} \notin \mathbb{F}_{q^{n}} \quad \text { for each } x \in \mathbb{F}_{q^{3 n}}^{*}
$$

then the set

$$
L_{U}=\left\{\left\langle f_{i, a, b}(x)+w x\right\rangle_{\mathbb{F}_{q^{2 n}}}: x \in \mathbb{F}_{q^{3 n}}^{*}\right\rangle
$$

turns out to be a scattered $\mathbb{F}_{q}$-linear of rank $3 n$ of the projective plane $\mathbb{P}=P G\left(\mathbb{F}_{q^{6 n}}, \mathbb{F}_{q^{2 n}}\right)=$ $P G\left(2, q^{2 n}\right)$

Proof. By Proposition 2.2, in order to prove the statement we have first to determine the solutions $x, y \in \mathbb{F}_{q^{3 n}}^{*}$ of Equations (5) and (6), with $f(x)=f_{i, a, b}(x)$ fulfilling Condition (16). With this choice Equation (5) becomes

$$
G(s):=b^{q^{2 n}+1} s^{q^{2 n+i}}-b^{q^{2 n}} a s^{q^{n+i}}+a^{q^{2 n}+1} s^{q^{i}}+A s=0,
$$

where $s=x y^{q^{2 n}}-y x^{q^{2 n}}$. By (16),$f_{i, a, b}(x) \neq 0$ for each $x \in \mathbb{F}_{q^{3 n}}^{*}$ and then $N_{q^{3 n} / q^{n}}(a) \neq$ $-N_{q^{3 n} / q^{n}}(b)$. Hence $G(s)=0$ if and only if $a^{q^{n}} G(s)+b^{q^{2 n}} G(s)^{q^{n}}=0$, i.e.

$$
\left(N_{q^{3 n} / q^{n}}(a)+N_{q^{3 n} / q^{n}}(b)\right) s^{q^{i}}+A b^{q^{2 n}} s^{q^{n}}+a^{q^{n}} A s=0 .
$$

Let $L:=N_{q^{3 n} / q^{n}}(a)+N_{q^{3 n} / q^{n}}(b)$ and note that by (16) $L \neq 0$. This means that if $s_{0}$ is a non-zero solution of (17), then $s_{0}$ satisfies the following equation

$$
\frac{b^{q^{2 n-i}} s_{0}^{q^{n-i}}+a^{q^{n-i}} s_{0}^{q^{3 n-i}}}{s_{0}}=\left(\frac{-L}{A}\right)^{q^{3 n-i}}
$$


i.e. there exists $s_{0} \in \mathbb{F}_{q^{3 n}}^{*}$ such that

$$
\frac{f_{n-i, b^{q^{2 n-i}}, a^{q^{n-i}}\left(s_{0}\right)}}{s_{0}} \in \mathbb{F}_{q^{n}}
$$

and hence

$$
\left(\frac{f_{n-i, b^{q^{2 n-i}}, a^{q^{n-i}}\left(s_{0}\right)}}{s_{0}}\right)^{q^{2 n}}=\frac{f_{n-i, b^{q^{n-i}}, a^{q^{3 n-i}}}\left(s_{0}^{q^{2 n}}\right)}{s_{0}^{q^{2 n}}} \in \mathbb{F}_{q^{n}} .
$$

Now, by Lemma 2.6 the maps $f_{i, a, b}(x) / x$ and $\bar{f}_{i, a, b}(x) / x$ have the same image and a direct calculation shows that

$$
\bar{f}_{i, a, b}=f_{n-i, b^{q^{n-i}}, a^{q^{3 n-i}}},
$$

hence by (16)

$$
\frac{f_{n-i, b^{q^{n-i}}, a^{q^{3 n-i}}(x)}}{x} \notin \mathbb{F}_{q^{n}} \quad \text { for each } x \in \mathbb{F}_{q^{3 n}}^{*}
$$

This means that Equation (17) only admits the zero solution, i.e. $s=x y^{q^{2 n}}-y x^{q^{2 n}}=0$, which implies $x=\alpha y$ for some $\alpha \in \mathbb{F}_{q^{n}}^{*}$. Now, from Equation (6) , substituting $f_{i, a, b}(x)=a x^{q^{i}}+b x^{q^{2 n+i}}$ and $x=\alpha y$, we get

$$
\left(\alpha^{q^{i}}-\alpha\right)\left(f_{i, a, b}(y)^{q^{2 n}} y-f_{i, a, b}(y) y^{q^{2 n}}\right)=0 .
$$

If $\alpha^{q^{i}} \neq \alpha$, we get from the previous equation

$$
f_{i, a, b}(y)^{q^{2 n}} y=f_{i, a, b}(y) y^{q^{2 n}}
$$

for some $y \in \mathbb{F}_{q^{3 n}}^{*}$, i.e. $f_{i, a, b}(y) / y \in \mathbb{F}_{q^{n}}$, a contradiction. Hence the element $\alpha \in \mathbb{F}_{q^{n}}^{*}$ is such that $\alpha^{q^{i}}=\alpha$, and since $\operatorname{gcd}(i, n)=1$, we get $\alpha \in \mathbb{F}_{q}^{*}$. As in Theorems 2.3 and 2.4, putting $x=\alpha y$, with $x, y \in \mathbb{F}_{q^{3 n}}^{*}$ and $\alpha \in \mathbb{F}_{q}^{*}$ in (7) we get $\lambda=\alpha \in \mathbb{F}_{q}^{*}$, proving the assertion by Proposition 2.2 ,

In what follows we will prove that if $q=2, i=1$ and $a=1$, then there exists at least an element $b \in \mathbb{F}_{q^{3 n}}^{*}$ such that Condition (16) is satisfied. To this end we need the following preliminary result. 
Lemma 2.8. Set $n>1$ and $q=2$, consider the function $H(t)=(1-t) / t^{j}$ defined in $\mathbb{F}_{q^{3 n}}^{*}$, where $j=\frac{q^{2 n+1}-1}{q-1}=2^{2 n+1}-1$. Then there exists at least an element $b \in \mathbb{F}_{2^{3 n}}^{*}$ not in the image of $H$ and such that $N_{2^{3 n} / 2^{n}}(b) \neq 1$.

Proof. First of all notice that $\left\{H(t) \mid t \in \mathbb{F}_{2^{3 n}}^{*}\right\}=\left\{t^{m}+t^{m-1} \mid t \in \mathbb{F}_{2^{3 n}}^{*}\right\}$, with $m=2^{3 n}-j=$ $2^{3 n}-2^{2 n+1}+1$. The function $\theta: \mathbb{F}_{2^{3 n}} \rightarrow \mathbb{F}_{2^{3 n}}$, defined by $\theta(x)=x^{q^{n-1}}$, is an automorphism of $\mathbb{F}_{2^{3 n}}$ and hence

$$
N_{2^{3 n} / 2^{n}}(x)=1 \Longleftrightarrow N_{2^{3 n} / 2^{n}}(\theta(x))=1
$$

Therefore

$$
\begin{aligned}
& \exists b \in \mathbb{F}_{2^{3 n}}^{*}: b \notin \operatorname{Im}(H), N_{2^{3 n} / 2^{n}}(b) \neq 1 \Longleftrightarrow \\
& \exists b \in \mathbb{F}_{2^{3 n}}^{*}: \quad b \notin \operatorname{Im}(\theta \circ H), N_{2^{3 n} / 2^{n}}(b) \neq 1 .
\end{aligned}
$$

We have that

$$
G(t)=(\theta \circ H)(t)=t^{\left(2^{3 n}-2^{2 n+1}+1\right) 2^{n-1}}+t^{\left(2^{3 n}-2^{2 n+1}\right) 2^{n-1}}=t^{2^{n}-1}+t^{2^{n-1}-1} .
$$

Since $n>1$ and $G(0)=G(1)=0, G(t)$ is not a permutation polynomial and it has degree $2^{n}-1$. Then by [20], its value set has size at most $2^{3 n}-\frac{2^{3 n}-1}{2^{n}-1}=2^{3 n}-\left(2^{2 n}+2^{n}+1\right)$. The number of elements of $\mathbb{F}_{2^{3 n}}$ having norm over $\mathbb{F}_{2^{n}}$ equal to 1 is exactly $2^{2 n}+2^{n}+1$. In the following we will prove that there exist at least $2^{n}+2$ elements in the value set of $G$ having norm over $\mathbb{F}_{2^{n}}$ equal to 1 . Note that an element having norm equal to 1 has the form $x^{2^{n}-1}$ for some $x \in \mathbb{F}_{2^{3 n}}^{*}$. Consider the curve $\mathcal{C}$ defined by

$$
f(x, y)=y^{2^{n}-1}+y^{2^{n-1}-1}+x^{2^{n}-1}=0 .
$$

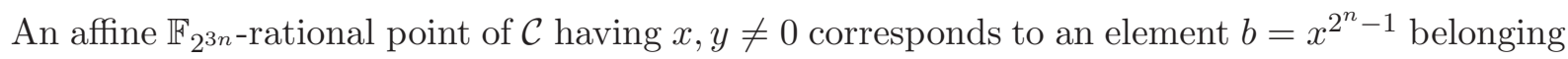
to the image of $G$ such that $N_{2^{3 n} / 2^{n}}(b)=1$. Intersecting the curve $\mathcal{C}$ with the lines $\ell_{t}: x=t y$ we get that the coordinates of the $\mathbb{F}_{2^{3 n}}$-rational points of $\mathcal{C}$ having $x, y \neq 0$ are of the form

$$
x=\frac{t}{\left(t^{2^{n}-1}-1\right)^{2^{2 n+1}}} \quad y=\frac{1}{\left(t^{2^{n}-1}-1\right)^{2^{2 n+1}}},
$$


where $t \in \mathbb{F}_{2^{3 n}} \backslash \mathbb{F}_{2^{n}}$. Hence $\mathcal{C}$ has exactly

$$
2^{3 n}-2^{n}
$$

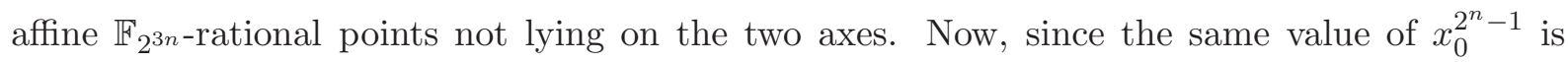
obtained $2^{n}-1$ times and since on the vertical line $x=x_{0}$ the curve $\mathcal{C}$ has at most $2^{n}-1$ points, we have that the same element in the image of $G$, with norm 1 , can be obtained from at most $\left(2^{n}-1\right)^{2}$ points of $\mathcal{C}$. Then there are at least

$$
\frac{2^{3 n}-2^{n}}{\left(2^{n}-1\right)^{2}}>2^{n}+2
$$

elements in the image of $G$ having norm equal to 1 . This proves that there exists at least an element $b \in \mathbb{F}_{2^{3 n}}^{*}$ not in the image of $H$ and of norm different from 1 .

Now, we are able to prove

Proposition 2.9. Let $f_{i, a, b}$ be the $\mathbb{F}_{q}$-linear map of $\mathbb{F}_{q^{3 n}}$ as defined in Proposition 2.7 and put $i=a=1$. If $q=2$, there exists at least one element $b \in \mathbb{F}_{2^{3 n}}^{*}$ such that

$$
\frac{f_{1,1, b}(x)}{x} \notin \mathbb{F}_{2^{n}} \quad \text { for each } x \in \mathbb{F}_{2^{3 n}}^{*}
$$

Proof. Taking $q=2$ and $i=a=1$ in $f_{i, a, b}(x)=a x^{2^{i}}+b x^{2^{2 n+i}}$, Condition (18) reads

$$
\frac{x^{2}+b x^{2^{2 n+1}}}{x}=x+b x^{2^{2 n+1}-1} \notin \mathbb{F}_{2^{n}} \quad \text { for each } x \in \mathbb{F}_{2^{3 n}}^{*}
$$

Let $g(x):=\frac{f_{1,1, b}(x)}{x}=x+b x^{2^{2 n+1}-1}$ for each $x \in \mathbb{F}_{2^{3 n}}^{*}$. Note that since $g(\eta x)=\eta g(x)$ for each $\eta \in \mathbb{F}_{q^{n}}$, Condition (19) is satisfied if $g(x) \neq 0$ and $g(x) \neq 1$ for each $x \in \mathbb{F}_{2^{3 n}}^{*}$. If there is an element $x_{0} \in \mathbb{F}_{q^{3 n}}^{*}$ such that $g\left(x_{0}\right)=1$, then the corresponding $b$ belongs to the image of the function $H$ defined in Lemma 2.8. If there is an element $x_{0} \in \mathbb{F}_{q^{3 n}}^{*}$ such that $g\left(x_{0}\right)=0$, then the corresponding $b$ has norm equal to 1 . By Lemma 2.8 there is an element $b_{0} \in \mathbb{F}_{2^{3 n}}^{*}$ not belonging to the image of $H$ and having norm different from 1 . This implies that Condition (19), for $b_{0}$, is satisfied and hence $f_{1,1, b_{0}}$ satisfies Condition (18). 
Putting together Propositions 2.7 and 2.9 we get the following

Theorem 2.10. For each integer $n>1$, the set

$$
L_{U}=\left\{\left\langle x^{2}+b x^{2^{2 n+1}}+x \omega\right\rangle_{\mathbb{F}_{2 n}}: x \in \mathbb{F}_{2^{3 n}}^{*}\right\}
$$

where $b \in \mathbb{F}_{2^{3 n}}^{*}$ with $N_{2^{3 n} / 2^{n}}(b) \neq 1$ and such that

$$
x+b x^{2^{2 n+1}-1} \notin \mathbb{F}_{2^{n}} \quad \text { for each } x \in \mathbb{F}_{2^{3 n}}^{*}
$$

is a scattered $\mathbb{F}_{2}$-linear set of the projective plane $P G\left(2,2^{2 n}\right)$ of rank $3 n$.

Remark 2.11. MAGMA computational results show that for $n=3$ and $q \in\{3,4,5\}$ there exist elements $b \in \mathbb{F}_{q^{3 n}}^{*}$ for which the functions $f_{1,1, b}$ satisfy Condition (18) yielding $\mathbb{F}_{q}$-scattered linear sets in $P G\left(2, q^{6}\right), q \in\{3,4,5\}$, of rank 9. However, taking Theorems 2.5 and 2.10 into account, the existence of a family of scattered $\mathbb{F}_{q}$-linear sets in $P G\left(2, q^{2 n}\right)$ for each $n \equiv 0$ mod 3 , $q \not \equiv 1 \bmod 3$ and $q>2$, remains an open problem.

\section{Constructions in $P G\left(r-1, q^{t}\right)$}

First of all we prove the following

Theorem 3.1. Let $\mathbb{P}=P G\left(\mathbb{V}, F_{q^{t}}\right)=P G\left(r-1, q^{t}\right)$ be a projective space and let

$$
\mathbb{V}=V_{1} \oplus_{\mathbb{F}_{q^{t}}} \cdots \oplus_{\mathbb{F}_{q^{t}}} V_{m}
$$

with $\operatorname{dim} V_{i}=s_{i} \geq 2$ and $i \in\{1, \ldots, m\}$. If $L_{U_{i}}$ is a scattered $\mathbb{F}_{q}$-linear set of $P G\left(V_{i}, \mathbb{F}_{q^{t}}\right)=$ $P G\left(s_{i}-1, q^{t}\right)$ then $L_{W}$, where

$$
W=U_{1} \oplus_{\mathbb{F}_{q}} \cdots \oplus_{\mathbb{F}_{q}} U_{m}
$$

is a scattered $\mathbb{F}_{q}$-linear set of $\mathbb{P}$.

Also, $L_{W}$ has maximum rank $\frac{r t}{2}$ if and only if each $L_{U_{i}}$ has maximum rank $\frac{s_{i} t}{2}$. 
Proof. Let $k_{i}$ be the rank of $L_{U_{i}}$. By Theorem $1.1 k_{i} \leq \frac{s_{i} t}{2}$ for each $i \in\{1, \ldots, m\}$. It is clear that $L_{W}$ is an $\mathbb{F}_{q}$-linear set of $\mathbb{P}$ of rank $\sum_{i=1}^{m} k_{i} \leq \sum_{i=1}^{m} \frac{s_{i} t}{2}=\frac{r t}{2}$. If $P:=\langle\underline{w}\rangle$ is a point of $L_{W}$ with weight grater than 1 , then there exist $\underline{w}^{\prime} \in W, \underline{w}^{\prime} \neq \underline{0}$, and $\lambda \in \mathbb{F}_{q^{t}} \backslash \mathbb{F}_{q}$ such that $\underline{w}=\lambda \underline{w}^{\prime}$. By (21), the vectors $\underline{w}$ and $\underline{w}^{\prime}$ can be uniquely written as

$$
\underline{w}=\underline{u}_{1}+\cdots+\underline{u}_{m} \quad \text { and } \quad \underline{w}^{\prime}=\underline{u}_{1}^{\prime}+\cdots+\underline{u}_{m}^{\prime}
$$

where $\underline{u}_{i}, \underline{u}_{i}^{\prime} \in U_{i}$ for each $i \in\{1, \ldots, m\}$. Taking $\underline{w}=\lambda \underline{w}^{\prime}$ and (20) into account, from the previous equalities we get $\underline{u}_{i}=\lambda \underline{u}_{i}^{\prime}$ for each $i \in\{1, \ldots, m\}$. Suppose that $j \in\{1, \ldots, m\}$ is the smallest number such that $\underline{u}_{j} \neq \underline{0}$. Then $\underline{u}_{j}=\lambda \underline{u}_{j}^{\prime}$, with $\underline{u}_{j} \in U_{j}$, and since $L_{U_{j}}$ is scattered we get $\lambda \in \mathbb{F}_{q}^{*}$, a contradiction. The last part is obvious.

The previous theorem can be naturally applied when $r$ is even by considering scattered $\mathbb{F}_{q^{-}}$ linear sets of rank $t$ on $\frac{r}{2}$ lines, say $\ell_{i}$, spanning the whole space $P G\left(r-1, q^{t}\right)$. In such a way we get a scattered $\mathbb{F}_{q}$-linear set in $P G\left(r-1, q^{t}\right)$ of rank $\frac{r t}{2}$. We will call this construction of type $(C 1)$. Some scattered linear sets reflecting this construction are those called of pseudoregulus type (see [13, Definitions 3.1 and 4.1]), for which each scattered linear set on $\ell_{i}$ is of pseudoregulus type (see [13, Remark 4.5]). Linear sets of pseudoregulus type have been also studied in [16], [10], [12] and to this family belongs the first explicit example of scattered linear sets obtained by Construction $(C 1)$ (see proof of [9, Thm. 2.5.5]). Also, from [13, Example $4.6(i)$ and $(i i)$ ] it is clear that, by using Construction $(C 1)$, we can also obtain scattered linear sets in $P G\left(r-1, q^{t}\right)$, $r$ even, of rank $\frac{r t}{2}$ which are not of pseudoregulus type.

\section{Proof of Theorem 1.2}

Putting together Theorems 2.3, 2.4 and 2.10 and Theorem 3.1, it follows that when $t$ is even and $r \geq 5$ we have several ways to construct scattered $\mathbb{F}_{q^{-}}$-linear sets in $P G\left(\mathbb{V}, F_{q^{t}}\right)=P G\left(r-1, q^{t}\right)$ of rank $\frac{r t}{2}$, by decomposing $\mathbb{V}$ as a direct sum over $\mathbb{F}_{q^{t}}$ of vector spaces of dimension 2 and 3 , 
proving in this way Theorem 1.2. Obviously, the greater is the integer $r$, the wider are these possible constructions.

Remark 3.2. From Theorem 1.1, each scattered $\mathbb{F}_{q^{-}}$-linear set of $P G\left(r-1, q^{2 n}\right)$ of rank $r n$ is a two-intersection set of the space with respect to the hyperplanes with intersection numbers

$\theta_{(r-2) n-1}(q)=\frac{q^{(r-2) n}-1}{q-1}$ and $\theta_{(r-2) n}(q)=\frac{q^{(r-2) n+1}-1}{q-1}$. Then, $L_{U}$ is a $\theta_{(r-2) n-1}(q)$-fold blocking set (with respect to hyperplanes) in $P G\left(r-1, q^{2 n}\right)$ ([3, Thm. 6.1]) and gives to rise two-weight linear codes and strongly regular graphs (see [5] and [3, Sec. 5]). As observed in [4], we want to stress that the parameters of these two-intersection sets are not new. Indeed, sets with the same parameters can be obtained by taking the disjoint union of $\frac{q^{n}-1}{q-1}$ Baer subgeometries in $P G\left(r-1, q^{2 n}\right)$ isomorphic to $P G\left(r-1, q^{n}\right)$. This set is called of type $I$ in [4]. Also in [4, Thm. 2.2], the authors show that a scattered $\mathbb{F}_{q}$-linear set of maximum rank cannot contain any Baer subgeometry of $P G\left(r-1, q^{2 n}\right)$ and hence the corresponding two-intersection set is not isomorphic to a set of type $I$.

\section{Small complete caps from maximum scattered linear sets}

Many links between the theory of linear sets and a large number of geometrical objects are known. Among them, two-intersection sets, blocking sets or multiple blocking sets, translation ovoids of polar spaces, translation spreads of the Cayley Generalized Hexagon $H(q)$. Also, linear sets are widely used in the construction of finite semifields. In this section we describe a connection between $F_{2}$-linear sets and another classical object in Finite Geometry: complete caps in Galois spaces. Such a connection is indeed fruitful; in fact, the results of the previous section on $F_{2}$-linear sets provide a solution, for spaces of even square order, to the long-standing problem of establishing whether the theoretical lower bound for the size of a complete cap is substantially sharp.

We first recall a Definition from [6, Sec. 2]. 
Definition 4.1. Let $q=2^{t}$ and let $G$ be an additive subgroup of $\mathbb{F}_{q}^{r}$. Let

$$
\mathcal{K}_{G}:=\left\{P_{v} \mid v \in G\right\} \subset A G(r, q),
$$

where $P_{v}$ is the affine point with coordinates $\left(a_{1}, a_{2}, \ldots, a_{r}\right)$ corresponding to the vector $v=$ $\left(a_{1}, a_{2}, \ldots, a_{r}\right) \in \mathbb{F}_{q}^{r}$. A translation cap is a cap in $A G(r, q)$ which coincides with $\mathcal{K}_{G}$ for some additive subgroup $G$ of $\mathbb{F}_{q}^{r}$.

Translation caps can be characterized as follows.

Theorem 4.2. [6, Lemma 2.1] For an additive subgroup $G$ of $\mathbb{F}_{q}^{r}$, $q$ even, the set $K_{G}$ is a translation cap if and only if any two non-zero distinct vectors in $G$ are $\mathbb{F}_{q}$-linearly independent.

Proposition 4.3. An $\mathbb{F}_{2}$-scattered linear set in $P G\left(r-1,2^{t}\right), t>1$, corresponds to a translation cap in $A G\left(r, 2^{t}\right)$ and viceversa.

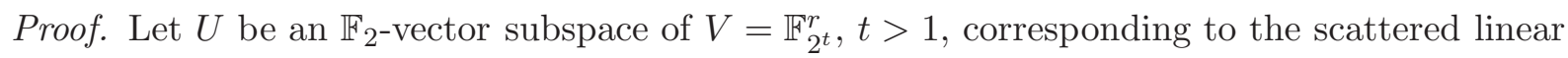
set $L_{U}$ in $P G\left(V, \mathbb{F}_{2^{t}}\right)$. Since $U$ in an additive subgroup of $V$, by Theorem $4.2, \mathcal{K}_{U}$ is a translation cap if and only if there no two distinct vectors in $U$ are $\mathbb{F}_{2^{t}}$-linearly dependent. This happens if and only all the elements of $U$ correspond to distinct points of $L_{U}$, that is $L_{U}$ is a scattered $\mathbb{F}_{2}$-linear set.

Let $\mathcal{S} \mathcal{L}$ and $\mathcal{T C}$ be the sets of all the scattered linear sets in $P G\left(r-1,2^{t}\right)$ and all the translation caps in $A G\left(r, 2^{t}\right)$. Form the previous theorem we can deduce the existence of a bijective function

$$
\varphi: \mathcal{S} \mathcal{L} \rightarrow \mathcal{T C}
$$

which sends $L_{U}$ to $\varphi\left(L_{U}\right)=\mathcal{K}_{U}$ for each $\mathbb{F}_{2}$-vector subspace $U$ of $V=\mathbb{F}_{2^{t}}^{r}$.

Proposition 4.4. Let $U_{1}$ and $U_{2}$ such that $\mathcal{K}_{U_{1}}$ and $\mathcal{K}_{U_{2}}$ are equivalent under the action of $A \Gamma L\left(r, 2^{t}\right)$. Then $\varphi^{-1}\left(\mathcal{K}_{U_{1}}\right)$ and $\varphi^{-1}\left(\mathcal{K}_{U_{2}}\right)$ are equivalent under the action of $\operatorname{P\Gamma L}\left(r, 2^{t}\right)$. 
Proof. Let $f \in A \Gamma L\left(r, 2^{t}\right)$ be such that $f\left(\mathcal{K}_{U_{1}}\right)=\mathcal{K}_{U_{2}}$. Then $f$ contains no translations, since it has to fix the 0 -vector. Then $f=M \tau$ with $M \in G L\left(r, 2^{t}\right)$ and $\tau \in A u t\left(\mathbb{F}_{2^{t}}\right)$. Let

$$
\mathcal{K}_{U_{1}}=\left\{0, P_{1}, P_{2}, \ldots, P_{n}\right\}, \quad \mathcal{K}_{U_{2}}=\left\{0, Q_{1}, Q_{2}, \ldots, Q_{n}\right\},
$$

with $f(0)=0$ and $f\left(P_{i}\right)=Q_{i}$. Also, let $\varphi^{-1}\left(\mathcal{K}_{U_{1}}\right)=\left\{\widetilde{P}_{1}, \widetilde{P}_{2}, \ldots, \widetilde{P}_{n}\right\}$ and $\varphi^{-1}\left(\mathcal{K}_{U_{2}}\right)=$ $\left\{\widetilde{Q}_{1}, \widetilde{Q}_{2}, \ldots, \widetilde{Q}_{n}\right\}$, with $\widetilde{P}_{i}=\lambda_{i} P_{i}, \widetilde{Q}_{i}=\mu_{i} Q_{i}$ and $\lambda_{i}, \mu_{i} \neq 0$ for all $i=1, \ldots, n$. Consider $g=\frac{1}{\operatorname{det}(M)} M \tau \in P \Gamma L\left(r, 2^{h}\right)$. Then

$$
\begin{gathered}
g\left(\widetilde{P}_{i}\right)=\left(\frac{1}{\operatorname{det}(M)} M \tau\right)\left(\widetilde{P}_{i}\right)=\left(\frac{1}{\operatorname{det}(M)} M \tau\right)\left(\lambda_{i} P_{i}\right)= \\
=\frac{\tau\left(\lambda_{i}\right)}{\operatorname{det}(M)}(M \tau)\left(P_{i}\right)=\frac{\tau\left(\lambda_{i}\right)}{\operatorname{det}(M)} Q_{i}=\frac{\tau\left(\lambda_{i}\right)}{\mu_{i} \operatorname{det}(M)} \widetilde{Q}_{i} .
\end{gathered}
$$

Then $g$ sends $\widetilde{P}_{i}$ to $\widetilde{Q}_{i}$ and $\varphi^{-1}\left(\mathcal{K}_{U_{1}}\right)$ is projectively equivalent to $\varphi^{-1}\left(\mathcal{K}_{U_{2}}\right)$.

By [6, Proposition 2.5] the maximum size of a translation cap in $A G(r, q), q=2^{t}$ and $t>1$, is $q^{\frac{r}{2}}$; if the bound is attained then the cap is said to be a maximal translation cap. We recall two further results from [6].

Lemma 4.5. [6, Proposition 2.8] If $\mathcal{K}_{G}$ is a maximal translation cap in $A G\left(r, 2^{t}\right)$, and $\mathcal{K}_{H}$ a maximal translation cap in $A G\left(\bar{r}, 2^{t}\right)$, then $\mathcal{K}_{G} \times \mathcal{K}_{H}$ is a maximal translation cap in $A G\left(r+\bar{r}, 2^{t}\right)$.

Lemma 4.6. (Doubling construction) [6, Corollary 2.12] If $\mathcal{K}_{G}$ is a maximal translation cap in $A G\left(r, 2^{t}\right)$, then $\mathcal{K}_{G \times\{0,1\}}$ is a complete cap in $A G\left(r+1,2^{t}\right)$.

We are now in a position to prove the key result of this section.

Proposition 4.7. Let $q=2^{t}, t$ even, and $n \geq 4$ even. If there exists a maximum scattered linear set in $P G(2, q)$, then there exists a complete cap in $A G(n, q)$ with size $2 q^{\frac{n-1}{2}}$.

Proof. Let $L$ be a maximum scattered $\mathbb{F}_{2}$-linear set of $P G\left(2,2^{t}\right)$. Since $t$ is even it has rank $\frac{3 t}{2}$. By Proposition 4.3 it is equivalent to a translation cap $\mathcal{K}$ in $A G\left(3,2^{t}\right)$ of size $2^{\frac{3 t}{2}}=\sqrt{q}^{3}$. Since 
the upper bound of [6. Proposition 2.5] is attained, $\mathcal{K}$ is a maximal translation cap in $A G\left(3,2^{t}\right)$. Let $n \geq 4$ even and consider in $A G\left(n-1,2^{t}\right)$ the following cap of size $q^{\frac{n-1}{2}}$ :

$$
\overline{\mathcal{K}}=\left\{\left(a, b, c, x_{1}, x_{1}^{2}, x_{2}, x_{2}^{2}, \ldots, x_{\frac{n-4}{2}}, x_{\frac{n-4}{2}}^{2}\right):(a, b, c) \in \mathcal{K}, x_{i} \in \mathbb{F}_{2^{t}}\right\}
$$

By Lemma 4.5, together with the fact that $\left.\left\{\left(x, x^{2}\right): x \in \mathbb{F}_{2^{t}}\right)\right\}$ is a translation cap in $A G\left(2,2^{t}\right)$, $\overline{\mathcal{K}}$ is a maximal translation cap in $A G\left(n-1,2^{t}\right)$. Now the cap

$$
\overline{\overline{\mathcal{K}}}=\left\{\left(a_{1}, \ldots, a_{n-1}, 0\right):\left(a_{1}, \ldots, a_{n-1}\right) \in \overline{\mathcal{K}}\right\} \cup\left\{\left(a_{1}, \ldots, a_{n-1}, 1\right):\left(a_{1}, \ldots, a_{n-1}\right) \in \overline{\mathcal{K}}\right\}
$$

is a complete translation cap in $A G\left(n, 2^{t}\right)$ of size $2 q^{\frac{n-1}{2}}$ by Lemma 4.6.

The existence of a complete cap in $A G(n, q)$ of size $2 q^{\frac{n-1}{2}}$, for $n \geq 4$ even and $q$ an even square, now follows from Theorems 2.3, 2.4, 2.10 and Proposition 4.7, Theorem 1.3 in Introduction is then proved.

Remark 4.8. For $q$ an even square and $n \geq 4$ even, the trivial lower bound for complete caps is substantially sharp not only in the affine space $A G(n, q)$ but also in the projective space $P G(n, q)$. In fact, it is possible to show that in $P G(2 k+4, q), k \geq 0$ there exists a complete cap

of size at most $3 q^{k+\frac{3}{2}}+4 q^{k+1}+3 \frac{q^{k+1}-1}{q-1}$ containing the translation cap of size $2 q^{k+\frac{3}{2}}$ obtained in Theorem 1.3. The lengthy and technical proof is similar to those of [6, Theorem 4.7] and [8. Propositions 2.5 and 5.3], where a complete translation cap in $A G(n, q)$ is extended to a complete cap in $P G(n, q)$.

\section{References}

[1] S. BALL: Polynomials in finite geoemtries, in "Surveys in Combinatorics 1999" (J.D. Lamb and D.A. Preece, Eds), London Math. Soc. Lectures Note Series, Vol. 267, pp. 17-35, Cambridge Univ. Press, Cambridge, UK, 1999. 
[2] S. Ball, A. Blokhuis and M. Lavrauw: Linear $(q+1)$-fold blocking sets in $P G\left(2, q^{4}\right)$, Finite Fields Appl. 6 n. 4 (2000), 294-301.

[3] A. Blokhuis and M. Lavrauw: Scattered spaces with respect to a spread in $P G(n, q)$, Geom. Dedicata 81 No.1-3 (2000), 231-243.

[4] A. Blokhuis and M. Lavrauw: On two-intersection sets with respect to hyperplanes in projective spaces, J. Comb. Theory, Ser. A, 99 No.2 (2002), 377-382.

[5] R. Calderbank and W.M. Kantor: The geometry of two-weight codes, Bull. London Math. Soc. 18 (1986), 97-122.

[6] M. Giulietti: Small complete caps in $\operatorname{PG}(N, q)$, q even, Journal of Combinatorial Designs 15(5) (2007), 420-436.

[7] M. Givlietti: The geometry of covering codes: small complete caps and saturating sets in Galois spaces, in Surveys in Combinatorics 2013 - London Mathematical Society Lecture Note Series 409, Cambridge University Press, 2013, pp. 51-90.

[8] M. Givlietti And F. Pasticci: Quasi-Perfect Linear Codes with Minimum Distance 4, IEEE Transactions on Information Theory 53(5) (2007), 1928-1935.

[9] M. Lavrauw: Scattered Spaces with respect to Spreads and Eggs in Finite Projective Spaces, Ph.D. Thesis, 2001.

[10] M. Lavrauw, G. Marino, O. Polverino and R. Trombetti: $\mathbb{F}_{q}$-pseudoreguli of $P G\left(3, q^{3}\right)$ and scattered semifields of order $q^{6}$, Finite Fields Appl., 17 (2011), 225-239.

[11] M. Lavraum and G. Van de Voorde: On linear sets on a projective line, Des. Codes Cryptogr. 56 (2010), 89-104. 
[12] M. Lavrauw and G. Van de Voorde: Scattered linear sets and pseudoreguli, The Electronic Journal of Comb. 20(1) (2013).

[13] G. Lunardon, G. Marino, O. Polverino and R. Trombetti: Maximum scattered linear sets of pseudoregulus type and the Segre Variety $\mathcal{S}_{n, n}$, J. Algebr. Comb., 39 (2014), 807-831.

[14] G. Lunardon and O. Polverino: Translation ovoids of orthogonal polar spaces, Forum Math., 16 (2004), 663-66.

[15] G. Marino and O. Polverino: On the nuclei of a finite semifield. Theory and applications of finite fields, Contemp. Math., 579, Amer. Math. Soc., Providence, RI (2012), 123-141.

[16] G. Marino, O. Polverino and R. Trombetti: On $\mathbb{F}_{q}$-linear sets of $P G\left(3, q^{3}\right)$ and semifields, J. Combin. Theory Ser. A 114 (2007), 769-788.

[17] F. Pambianco and L. Storme: Small complete caps in spaces of even characteristic, J. Combin. Theory Ser. A 75(1) (1996), 70-84.

[18] O. Polverino: Linear sets in Finite Projective Spaces, Discrete Math., 310 (2010), 30963107.

[19] B. SEgRE: On complete caps and ovaloids in three-dimensional Galois spaces of characteristic two, Acta Arith., 5 (1959), 315-332.

[20] G. Turnwald: A new criterion for permutation polynomials, Finite Fields Appl. 1 (1995), $64-82$.

Daniele Bartoli and Massimo Giulietti

Dipartimento di Matematica e Informatica, 
Università degli Studi di Perugia,

I-06123 Perugia, Italy

daniele.bartoli@unipg.it,massimo.giulietti@unipg.it

Giuseppe Marino and Olga Polverino

Dipartimento di Matematica e Fisica,

Seconda Università degli Studi di Napoli,

I-81100 Caserta, Italy

giuseppe.marino@unina2.it,olga.polverino@unina2.it 